\title{
On the $p$-Biharmonic Operator with Critical Sobolev Exponent and Nonlinear Steklov Boundary Condition
}

\author{
Abdelouahed El Khalil, ${ }^{1}$ My Driss Morchid Alaoui, ${ }^{2}$ and Abdelfattah Touzani ${ }^{2}$ \\ ${ }^{1}$ Department of Mathematics and Statistics, College of Science, Al-Imam Mohammad Ibn Saud Islamic University (IMSIU), \\ P.O. Box 90950, Riyadh 11623, Saudi Arabia \\ ${ }^{2}$ Department of Mathematics, Faculty of Sciences Dhar-Mahraz, University Sidi Mohamed Ben Abdellah, \\ P.O. Box 1796, Atlas-Fez, Fez 30000, Morocco
}

Correspondence should be addressed to Abdelouahed El Khalil; alakhalil@imamu.edu.sa

Received 26 November 2013; Accepted 30 January 2014; Published 19 March 2014

Academic Editor: Patrick Guidotti

Copyright (C) 2014 Abdelouahed El Khalil et al. This is an open access article distributed under the Creative Commons Attribution License, which permits unrestricted use, distribution, and reproduction in any medium, provided the original work is properly cited.

We show that this operator possesses at least one nondecreasing sequence of positive eigenvalues. A direct characterization of the principal eigenvalue (the first one) is given that we apply to study the spectrum of the $p$-biharmonic operator with a critical Sobolev exponent and the nonlinear Steklov boundary conditions using variational arguments and trace critical Sobolev embedding.

\section{Introduction}

Let $\Omega$ be a smooth bounded domain in $\mathbb{R}^{N}$. Consider the fourth-order nonlinear Steklov boundary eigenvalue problem

$$
\begin{aligned}
\Delta_{p}^{2} u=|u|^{p^{*}-2} u \quad \text { in } \Omega, & \\
|\Delta u|^{p-2} \Delta u & =\lambda\left|\frac{\partial u}{\partial n}\right|^{p-2} \frac{\partial u}{\partial n} \quad \text { on } \Gamma \quad\left(E_{p}\right)_{\lambda} \\
u & \in W^{2, p}(\Omega) \cap W_{0}^{1, p}(\Omega),
\end{aligned}
$$

where $p^{*}$ is the critical Sobolev exponent defined by

$$
p^{*}=\frac{N p}{N-2 p} \quad \text { with } 2<2 p<N .
$$

Here $\lambda$ is a real parameter which plays the role of an eigenvalue.

$\Delta_{p}^{2} u:=\Delta\left(|\Delta u|^{p-2} \Delta u\right)$ is the operator of fourth order called the $p$-biharmonic operator. For $p=2$, the linear operator $\Delta_{2}^{2}=\Delta^{2}=\Delta . \Delta$ is the iterated Laplacian that multiplied with positive constant appears often in NavierStokes equations as being a viscosity coefficient. Its reciprocal operator, denoted by $\left(\Delta^{2}\right)^{-1}$, is celebrated Green's operator [1].

The nonlinear boundary condition describes a nonlinear flux through the boundary $\Gamma$ which depends on the solution itself and its normal derivation. Here $\partial u / \partial n$ denotes the outer normal derivative of $u$ on $\Gamma$ defined by $\partial u / \partial n=\nabla u \cdot \vec{n}$.

Notice that the biharmonic equation $\Delta^{2} u=0$, corresponding to $p=2$, is a partial differential equation of fourth order which appears in quantum mechanics and in the theory of linear elasticity modeling Stokes' flows. It is well known that elliptic problems with eigenvalues in the boundary conditions are usually called Steklov problems from their first appearance in [2]. For the fourth-order Steklov eigenvalue problems, the first eigenvalue plays a crucial role in the positivity preserving property for the biharmonic operator under conditions $u=0, \Delta u-\lambda(\partial u / \partial n)=0$ on $\Gamma$ (see [3]). In [4], the authors investigated the bound for the first eigenvalue on the plane square and proved that the first eigenvalue is simple and its eigenfunction does not change sign. The authors of $[5,6]$ studied the spectrum of a fourthorder Steklov eigenvalue problem on a bounded domain in 
$\mathbb{R}^{N}$ and gave the explicit form of the spectrum in the case where the domain is a ball. Let us mention that the spectrum of the fourth order Steklove has been completely determined by Ren and Yang [7] in the case $p=2$, using the theory of completely continuous operators.

It is already evident from the well-studied second-order case that nonlinear equations with critical growth terms present highly interesting phenomena concerning the existence and nonexistence. For the fourth-order equations is more challenging, since the techniques depend strongly on the imposed boundary conditions.

For the case of $p=2, p^{*}=2 N /(N-4)$, with $N \geq 5$, the problem $\left(E_{p=2}\right)_{\lambda}$ becomes linear on the right side and it is studied by Berchio et al. in [8].

It is well known that fourth-order elliptic problems arise in many applications, such as microelectromechanical system, in thin film theory, nonlinear surface diffusion on solids, interface dynamics, flow in Hele-Shaw cells, phase field models of multi-phase systems, and the deformation of a nonlinear elastic beam; see, for example, $[9,10]$, for more details.

In the nonlinear cases of $p \neq 2$, problems governed by $p$ biharmonic operators attracted growing interest, and figure in variety of applications, where this operator is used to control the nonlinearity artificial viscosity of diffusion surface of non-Newtonian fluids [11].

Recently, El Khalil et al. in [12] proved that the following nonlinear boundary problem:

$$
\begin{array}{r}
\Delta_{p}^{2} u=|u|^{p-2} u \quad \text { in } \Omega \\
-\frac{\partial}{\partial n}\left(|\Delta u|^{p-2} \Delta u\right)=\lambda|u|^{p-2} u \quad \text { on } \Gamma \\
u \in W^{2, p}(\Omega) \cap W_{0}^{1, p}(\Omega),
\end{array}
$$

has at least one nondecreasing sequence of positive eigenvalues $\left(\lambda_{k}\right)_{k \geq 1}$.

In this paper, we use a variational technique to prove the existence of a sequence of positive eigenvalues of problem $\left(E_{p}\right)_{\lambda}$.

To present our result concerning $\left(E_{p}\right)_{\lambda}$, we consider the homogenous problem

$$
\begin{aligned}
\Delta_{p}^{2} u & =0 \quad \text { in } \Omega \\
|\Delta u|^{p-2} \Delta u & =\lambda\left|\frac{\partial u}{\partial n}\right|^{p-2} \frac{\partial u}{\partial n} \quad \text { on } \Gamma \quad\left(E_{p}\right)_{\lambda}^{\prime} \\
u & \in W^{2, p}(\Omega) \cap W_{0}^{1, p}(\Omega) .
\end{aligned}
$$

Throughout this paper, we use the notation $\mathbb{W}_{p}(\Omega):=$ $W^{2, p}(\Omega) \cap W_{0}^{1, p}(\Omega)$.

\section{Definitions and Preliminaries}

Definition 1. One says that a function $u \in \mathbb{W}_{p}(\Omega)$ is weak solution of $\left(E_{p}\right)_{\lambda}^{\prime}$ if

$$
\int_{\Omega}|\Delta u|^{p-2} \Delta u \Delta v d x=\lambda \int_{\Gamma}\left|\frac{\partial u}{\partial n}\right|^{p-2} \frac{\partial u}{\partial n} \frac{\partial v}{\partial n} d s \quad \forall v \in \mathbb{W}_{p}(\Omega) .
$$

If $u$ is not identically zero, then one says that $\lambda$ is an eigenvalue of $\left(E_{p}\right)_{\lambda}^{\prime}$ corresponding to the eigenfunction $u$.

The main objective of this work is to show that problem $\left(E_{p}\right)_{\lambda}^{\prime}$ has at least one nondecreasing sequence positive eigenvalues $\left(\lambda_{k}\right)_{k \geq 1}$, by using a variational technique based on mini-max theory on $C^{1}$-manifolds [13]. In fact, we give a direct characterization of $\lambda_{k}$ involving a mini-max argument over sets of genus greater than $k$.

We set

$$
\lambda_{1}=\inf \left\{\|\Delta v\|_{p}^{p}, v \in \mathbb{W}_{p}(\Omega) ; \int_{\Gamma}\left|\frac{\partial u}{\partial n}\right|^{p} d s=1\right\},
$$

where $\|\Delta \mathrm{v}\|_{p}=\left(\int_{\Omega}|\Delta \mathrm{v}|^{p}\right)^{1 / p}$ denoted by $\|\Delta .\|_{p}$ is a norm on $\mathbb{W}_{p}(\Omega)$. Let us notice that $\mathbb{W}_{p}(\Omega)$ equipped with this norm is a uniformly convex Banach space for $1<p<+\infty$. The norm $\|\Delta .\|_{p}$ is uniformly equivalent on $W_{0}^{2, p}(\Omega)$ to the usual norm of $W_{0}^{2, p}(\Omega)$. Indeed, in [14] the scalar $p$-polyharmonic operators $\Delta_{p}^{L}$, which coincide to the $p$-biharmonic for $L=2$, were recently introduced for all orders $L$ and independently in [15] only for $L$ even. The norms are proved to be equivalent; see also the vectorial case treated in [16]. The proof of the equivalence comes from the Poincar and Calderón-Zygmund type inequalities.

For reader's convenience, we give below the proof of the equivalence between the standard Sobolev space norm and the norm $\|\Delta .\|_{p}$. For that, consider the classical Dirichlet problem for the famous Poisson's equation (see [17]):

$$
\begin{gathered}
-\Delta u=f \quad \text { in } \Omega, \\
u=0 \quad \text { on } \Gamma .
\end{gathered}
$$

Let us denote by $\|u\|_{p}=\left(\int_{\Omega}|u|^{p} d x\right)^{1 / p}$ the norm in $L^{p}(\Omega)$ and by $\|u\|_{2, p}=\left(\|\Delta u\|_{p}^{p}+\|u\|_{p}^{p}\right)^{1 / p}$ the norm in $\mathbb{W}_{p}(\Omega)$.

If $T$ is the inverse operator of $-\Delta: \mathbb{W}_{p}(\Omega) \rightarrow L^{p}(\Omega)$, it is well known that (5) is uniquely solvable in $\mathbb{W}_{p}(\Omega)$ for all $f \in L^{p}(\Omega)$ and for any $\left.p \in\right] 1,+\infty[$. Moreover, the operator solution

$$
T: L^{p}(\Omega) \longrightarrow \mathbb{W}_{p}(\Omega)
$$

satisfies the elliptic regularity estimation (continuity)

$$
\|T f\|_{2, p} \leq C_{p}\|f\|_{p} .
$$

This allows us to say the following. 
If $f \in L^{p}(\Omega)$ and $u \in W_{0}^{1, p}(\Omega)$ is a solution of (5), then $-\Delta u \in L^{p}(\Omega), u=0$ on $\Gamma$ and $u \in \mathbb{W}_{p}(\Omega)$. Moreover,

$$
\|T(-\Delta u)\|_{2, p} \leq C_{p}\|\Delta u\|_{p} .
$$

That is,

$$
\|u\|_{2, p} \leq C_{p}\|\Delta u\|_{p}
$$

for a suitable positive constant $C_{p}$ independent of $f$.

On the other hand, it is easy to remark that

$$
\|\Delta u\|_{p} \leq\|u\|_{2, p} .
$$

By the Closed Graph Theorem, we conclude that $u \rightarrow$ $\|\Delta u\|_{p}=\left(\int_{\Omega}|\Delta u|^{p} d x\right)^{1 / p}$ is equivalent to the norm induced by $W^{2, p}(\Omega)$.

We see that the value defined in (4) can be written as

$$
\lambda_{1}=\inf _{u \in \mathbb{W}_{p}(\Omega), u \neq 0} \frac{\int_{\Omega}|\Delta u|^{p} d x}{\int_{\Gamma}|\partial u / \partial n|^{p} d s} .
$$

Finally, let us point out that the problem $\left(E_{p}\right)_{\lambda}^{\prime}$ is naturally well defined taking in account the trace embedding $\mathbb{W}_{p}(\Omega) \hookrightarrow L^{p}(\partial \Omega)$ introduced in $[12]$.

Definition 2. Let $X$ be a real reflexive Banach space and let $X^{*}$ stand for its dual with respect to the pairing $\langle\cdot, \cdot\rangle$. We shall deal with mappings $T$ acting from $X$ into $X^{*}$. The strong convergence in $X$ (and in $X^{*}$ ) is denoted by $\rightarrow$ and the weak convergence by $\rightarrow$. T is said to belong to the class $\left(S^{+}\right)$if for any sequence $u_{n}$ in $X$ converging weakly to $u \in X$ and $\lim \sup _{n \rightarrow+\infty}\left\langle\Delta_{p}^{2} u_{n}, u_{n}-u\right\rangle \leq 0$ it follows that $u_{n}$ converges strongly to $u$ in $X$. We write $T \in\left(S^{+}\right)$.

Consider now the following two functionals defined on $\mathbb{W}_{p}(\Omega)$ :

$$
\Phi(u)=\frac{1}{p} \int_{\Omega}|\Delta u|^{p} d x, \quad \varphi(u)=\frac{1}{p} \int_{\Gamma}\left|\frac{\partial u}{\partial n}\right|^{p} d s,
$$

and set $\mathfrak{M}=\left\{u \in \mathbb{W}_{p}(\Omega) ; p \varphi(u)=1\right\}$.

Lemma 3. One has the following statements.

(i) $\Phi$ and $\varphi$ are even and of class $C^{1}$ on $\mathbb{W}_{p}(\Omega)$.

(ii) $\mathfrak{M}$ is a closed $C^{1}$-manifold.

Proof. It is clear that $\varphi$ and $\Phi$ are even and of class $C^{1}$ on $\mathbb{W}_{p}(\Omega)$ and $\mathfrak{M}=\varphi^{-1}\{1 / p\}$. Therefore $\mathfrak{M}$ is closed. The derivative operator $\varphi^{\prime}$ satisfies $\varphi^{\prime}(u) \neq 0$ for all $u \in \mathfrak{M}$ (i.e., $\varphi^{\prime}(u)$ is onto for all $u \in \mathfrak{M}$ ), so $\varphi$ is a submersion; then $\mathfrak{M}$ is a $C^{1}$-manifold.

Remark 4. Observe that $J: \mathbb{W}_{p}(\Omega) \rightarrow\left(\mathbb{W}_{p}(\Omega)\right)^{\prime}$ defined by

$$
J(u)= \begin{cases}\|\Delta u\|_{p}^{2-p} \Delta_{p}^{2} u & \text { if } u \neq 0 \\ 0 & \text { if } u=0\end{cases}
$$

is the duality mapping of $\mathbb{W}_{p}(\Omega)$ associated with the norm $\|\Delta .\|_{p}$.
The following lemma is the key of our result related to the existence.

\section{Lemma 5. One has the following statements.}

(i) $\varphi^{\prime}$ is completely continuous.

(ii) The functional $\Phi$ satisfies the Palais-Smale condition on $\mathfrak{M}$, that is, for $\left\{u_{n}\right\} \subset \mathfrak{M}$ if $\left\{\Phi\left(u_{n}\right)\right\}_{n}$ is bounded and

$$
\epsilon_{n}:=\Phi^{\prime}\left(u_{n}\right)-g_{n} \varphi^{\prime}\left(u_{n}\right) \longrightarrow 0 \text { as } n \longrightarrow+\infty,
$$

where $g_{n}=\left\langle\Phi^{\prime}\left(u_{n}\right), u_{n}\right\rangle /\left\langle\varphi^{\prime}\left(u_{n}\right), u_{n}\right\rangle$. Then $\left\{u_{n}\right\}_{n \geq 1}$ has a convergent subsequence in $\mathbb{W}_{p}(\Omega)$.

Proof. Let $u, v \in \mathbb{W}_{p}(\Omega)$. We have

$$
\left\langle\varphi^{\prime}(u), v\right\rangle=\int_{\Gamma}\left|\frac{\partial u}{\partial n}\right|^{p-2} \frac{\partial u}{\partial n} \frac{\partial v}{\partial n} d s .
$$

Then

$$
\left|\left\langle\varphi^{\prime}(u), v\right\rangle\right| \leq \int_{\Gamma}\left|\frac{\partial u}{\partial n}\right|^{p-1} \frac{\partial v}{\partial n} d s .
$$

By applying Hölder's inequality, we obtain

$$
\left|\left\langle\varphi^{\prime}(u), v\right\rangle\right| \leq\left\|\frac{\partial u}{\partial n}\right\|_{L^{p^{\prime}(\Gamma)}}^{p-1}\left\|\frac{\partial v}{\partial n}\right\|_{L^{p}(\Omega)},
$$

where $p$ and $p^{\prime}$ are conjugate by the equality $p p^{\prime}=p+p^{\prime}$. Therefore

$$
\left|\left\langle\varphi^{\prime}(u), v\right\rangle\right| \leq K\|\Delta u\|_{p^{\prime}}^{p-1}\|\Delta v\|_{p}
$$

Hence

$$
\left\|\varphi^{\prime}(u)\right\|_{*} \leq K\|\Delta u\|_{p^{\prime}}^{p-1},
$$

where $K$ is the constant given by the embedding of $\mathbb{W}_{p}(\Omega)$ in $L^{p}(\Gamma)$. Here $\|\cdot\|_{*}$ is the dual norm associated with $\|\Delta .\|_{p}$.

Now, by the definition of $\Phi$ we have that $\left\|\Delta\left(u_{n}\right)\right\|_{p}$ is bounded in $\mathbb{R}$.

Thus, without loss of generality, we can assume that $u_{n}$ converges weakly in $\mathbb{W}_{p}(\Omega)$ for some function $u \in \mathbb{W}_{p}(\Omega)$ and $\left\|\Delta\left(u_{n}\right)\right\|_{p} \rightarrow \delta$. For the rest we distinguish two cases.

If $\delta=0$, then $u_{n}$ converges strongly to 0 in $\mathbb{W}_{p}(\Omega)$.

If $\delta \neq 0$, then let us prove that

$$
\limsup _{n \rightarrow \infty}\left\langle\Delta_{p}^{2} u_{n}, u_{n}-u\right\rangle \leq 0
$$

Indeed, notice that

$$
\left\langle\Delta_{p}^{2} u_{n}, u_{n}-u\right\rangle=\left\|\Delta u_{n}\right\|_{p}^{p}-\left\langle\Delta_{p}^{2} u_{n}, u\right\rangle .
$$

Applying $\epsilon_{n}$ of (14) to $u$, we deduce that

$$
\theta_{n}:=\left\langle\Delta_{p}^{2} u_{n}, u\right\rangle-g_{n}\left\langle\varphi^{\prime}\left(u_{n}\right), u\right\rangle \longrightarrow 0, \quad \text { as } n \longrightarrow \infty .
$$


Thus

$$
\theta_{n}:=\left\langle\Delta_{p}^{2} u_{n}, u\right\rangle-\epsilon_{n} \cdot\left\langle\varphi^{\prime}\left(u_{n}\right), u\right\rangle \longrightarrow 0 \text { as } n \longrightarrow \infty .
$$

Therefore

$$
\begin{aligned}
& \left\langle\Delta_{p}^{2} u_{n}, u_{n}-u\right\rangle \\
& \quad=\left\|\Delta u_{n}\right\|^{p}-\theta_{n}-\left(\frac{\left\langle\Phi^{\prime}\left(u_{n}\right), u_{n}\right\rangle}{\left\langle\varphi^{\prime}\left(u_{n}\right), u_{n}\right\rangle}\right) \cdot\left\langle\varphi^{\prime}\left(u_{n}\right), u\right\rangle .
\end{aligned}
$$

That is,

$$
\begin{aligned}
& \left\langle\Delta_{p}^{2} u_{n}, u_{n}-u\right\rangle \\
& =\frac{\left\|\Delta u_{n}\right\|_{p}^{p}}{\left\langle\varphi^{\prime}\left(u_{n}\right), u_{n}\right\rangle}\left(\left\langle\varphi^{\prime}\left(u_{n}\right), u_{n}\right\rangle-\left\langle\varphi^{\prime}\left(u_{n}\right), u\right\rangle\right)-\theta_{n} .
\end{aligned}
$$

On the other hand, from Lemma $5, \varphi^{\prime}$ is completely continuous. Thus

$$
\begin{aligned}
\varphi^{\prime}\left(u_{n}\right) & \longrightarrow \varphi^{\prime}(u), \\
\left\langle\varphi^{\prime}\left(u_{n}\right), u_{n}\right\rangle & \longrightarrow\left\langle\varphi^{\prime}(u), u\right\rangle .
\end{aligned}
$$

Then

$$
\begin{aligned}
\left|\left\langle\varphi^{\prime}\left(u_{n}\right), u_{n}\right\rangle-\left\langle\varphi^{\prime}\left(u_{n}\right), u\right\rangle\right| \\
\leq\left|\left\langle\varphi^{\prime}\left(u_{n}\right), u_{n}\right\rangle-\left\langle\varphi^{\prime}(u), u\right\rangle\right| \\
\quad+\left|\left\langle\varphi^{\prime}\left(u_{n}\right), u\right\rangle-\left\langle\varphi^{\prime}(u), u\right\rangle\right| .
\end{aligned}
$$

It follows that

$$
\begin{aligned}
\left|\left\langle\varphi^{\prime}\left(u_{n}\right), u_{n}\right\rangle-\left\langle\varphi^{\prime}\left(u_{n}\right), u\right\rangle\right| & \\
\leq & \left|\left\langle\varphi^{\prime}\left(u_{n}\right), u_{n}\right\rangle-\left\langle\varphi^{\prime}(u), u\right\rangle\right| \\
& +\left\|\varphi^{\prime}\left(u_{n}\right)-\varphi^{\prime}(u)\right\|_{*}\|\Delta u\|_{p} .
\end{aligned}
$$

This implies that

$$
\left\langle\varphi^{\prime}\left(u_{n}\right), u_{n}\right\rangle-\left\langle\varphi^{\prime}\left(u_{n}\right), u\right\rangle \longrightarrow 0, \quad \text { as } n \longrightarrow \infty .
$$

Combining with the above equalities, we obtain

$$
\begin{aligned}
& \limsup _{n \rightarrow+\infty}\left\langle\Delta_{p}^{2} u_{n}, u_{n}-u\right\rangle \\
& \quad \leq \frac{\delta^{p}}{\left\langle\varphi^{\prime}(u), u\right\rangle} \limsup _{n \rightarrow \infty}\left(\left\langle\varphi^{\prime}\left(u_{n}\right), u_{n}\right\rangle-\left\langle\varphi^{\prime}\left(u_{n}\right), u\right\rangle\right) .
\end{aligned}
$$

We deduce

$$
\limsup _{n \rightarrow \infty}\left\langle\Delta_{p}^{2} u_{n}, u_{n}-u\right\rangle \leq 0
$$

We can write $\Delta_{p}^{2} u_{n}=\left\|\Delta u_{n}\right\|_{p}^{p-2} J\left(u_{n}\right)$, since $\left\|\Delta u_{n}\right\|_{p} \neq 0$ for $n$ large enough. Therefore

$$
\limsup _{n \rightarrow \infty}\left\langle\Delta_{p}^{2} u_{n}, u_{n}-u\right\rangle=\delta^{p-2} \limsup _{n \rightarrow \infty}\left\langle J u_{n}, u_{n}-u\right\rangle .
$$

According to (31), we conclude that

$$
\limsup _{n \rightarrow \infty}\left\langle J u_{n}, u_{n}-u\right\rangle \leq 0
$$

where $J$ is the duality mapping defined in Remark 4 . Thus it satisfies the condition $S^{+}$given in [18]. Therefore, $u_{n} \rightarrow u$ strongly in $\mathbb{W}_{p}(\Omega)$.

This achieves the proof of the lemma.

\section{Main Results}

Set

$$
\Gamma_{k}=\{K \subset \mathfrak{M}: K \text { symmetric, compact and } \gamma(K) \geq k\},
$$

where $\gamma(K)=k$ is the genus of $k$, that is, the smallest integer $k$ such that there exists an odd continuous map from $K$ to $\mathbb{R}^{k} \backslash\{0\}$.

Let us now state our first main result of this paper using mini-max theory; we have our main result formulated as follows.

Theorem 6. For any integer $k \in \mathbb{N}^{*}$,

$$
\lambda_{k}:=\inf _{K \in \Gamma_{k}} \max _{u \in K} p \Phi(u)
$$

is a critical value of $\Phi$ restricted on $\mathfrak{M}$. More precisely, there exist $u_{k} \in K$, such that

$$
\lambda_{k}=p \Phi\left(u_{k}\right)=\sup _{u \in K} p \Phi(u)
$$

and $u_{k}$ is a solution of $\left(E_{p}\right)_{\lambda}^{\prime}$ associated with positive eigenvalue $\lambda_{k}$. Moreover,

$$
\lambda_{k} \longrightarrow \infty, \quad \text { as } k \longrightarrow \infty \text {. }
$$

Proof. We only need to prove that, for any $k \in \mathbb{N}^{*}, \Gamma_{k} \neq \emptyset$ and the limit (37). Indeed, since $\mathbb{W}_{p}(\Omega)$ is separable, there exists $\left(e_{i}\right)_{i \geq 1}$ linearly dense in $W_{p}(\Omega)$ such that supp $e_{i} \cap \operatorname{supp} e_{j}=\emptyset$ if $i \neq j$. We may assume that $e_{i} \in \mathfrak{M}$ (if not, we take $e_{i}^{\prime}=$ $\left.e_{i} / \varphi\left(e_{i}\right)\right)$.

Let now $k \in \mathbb{N}^{*}$ and denote that

$$
F_{k}=\operatorname{span}\left\{e_{1}, e_{2}, \ldots, e_{k}\right\}
$$

Clearly, $F_{k}$ is a vector subspace with $\operatorname{dim} F_{k}=k$.

If $v \in F_{k}$, then there exist $\alpha_{1}, \ldots \alpha_{k}$ in $\mathbb{R}$ such that $v=$ $\sum_{i=1}^{k} \alpha_{i} e_{i}$. Thus

$$
\varphi(v)=\sum_{i=1}^{k}\left|\alpha_{i}\right|^{p} \varphi\left(e_{i}\right)=\frac{1}{p} \sum_{i=1}^{k}\left|\alpha_{i}\right|^{p} .
$$


It follows that the map

$$
v \longmapsto(p \varphi(v))^{1 / p}:=\|v\|
$$

defines a norm on $F_{k}$. Consequently, there is a constant $c>0$ such that

$$
c\|\Delta u\|_{p} \leq\|v\| \leq \frac{1}{c}\|\Delta u\|_{p}
$$

This implies that the set

$$
V=F_{k} \cap\left\{v \in \mathbb{W}_{p}(\Omega): p \varphi(v) \leq 1\right\}
$$

is bounded, since $V \subset B(0,1 / c)$, where

$$
B\left(0, \frac{1}{c}\right)=\left\{u \in \mathbb{W}_{p}(\Omega), \text { such that }\|\Delta u\| \leq \frac{1}{c}\right\} .
$$

Thus, $V$ is a symmetric bounded neighborhood of $0 \in F_{k}$. Moreover, $F_{k} \cap \mathfrak{M}$ is a compact set.

By Proposition 2.3 in [13], we conclude that $\gamma\left(F_{k} \cap \mathfrak{M}\right)=k$ and then we obtain finally that $\Gamma_{k} \neq \emptyset$.

This completes the proof of the theorem.

Now, we claim that

$$
\lambda_{k} \longrightarrow \infty, \quad \text { as } k \longrightarrow \infty
$$

Let $\left(e_{n}, e_{j}^{*}\right)_{n, j}$ be a biorthogonal system such that $e_{n} \in \mathbb{W}_{p}(\Omega)$ and $e_{j}^{*} \in\left(\mathbb{W}_{p}(\Omega)\right)^{\prime}$, the $e_{n}$ are linearly dense in $\mathbb{W}_{p}(\Omega)$, and the $e_{j}^{*}$ are total for the dual $\left(\mathbb{W}_{p}(\Omega)\right)^{\prime}$.

For $k \in \mathbb{N}^{*}$, set

$$
F_{k}=\operatorname{span}\left\{e_{1}, \ldots, e_{k}\right\}, \quad F_{k}^{\perp}=\operatorname{span}\left\{e_{k+1}, e_{k+2}, \ldots\right\} .
$$

We have, for any $K \in \Gamma_{k}, K \cap F_{k}^{\perp} \neq \emptyset$. Thus

$$
t_{k}:=\inf _{K \in \Gamma_{k}} \sup _{u \in K \cap F_{k}^{\perp}} p \Phi(u) \longrightarrow \infty, \quad \text { as } k \longrightarrow \infty
$$

Indeed, if not, for $k$ is large, there exists $u_{k} \in F_{k-1}^{\perp}$ with $\left\|u_{k}\right\|_{p}=1$ such that

$$
t_{k} \leq p \Phi\left(u_{k}\right) \leq M
$$

for some $M>0$ independent of $k$. Thus $\left\|\Delta u_{k}\right\|_{p} \leq M$. This implies that $\left(u_{k}\right)_{k}$ is bounded in $\mathbb{W}_{p}(\Omega)$. For a subsequence of $\left\{u_{k}\right\}$, if necessary, we can assume that $\left\{u_{k}\right\}$ converges weakly in $\mathbb{W}_{p}(\Omega)$ and strongly in $L^{p}(\Omega)$. By our choice of $F_{k-1}^{\perp}$, we have $u_{k} \rightarrow 0$ weakly in $\mathbb{W}_{p}(\Omega)$, because $\left\langle e_{n}^{*}, e_{k}\right\rangle=0$, for any $k \geq n$. This contradicts the fact that $\left\|u_{k}\right\|_{p}=1$ for all $k$. Since $\lambda_{k} \geq t_{k}$ the claim is proved. This achieved the proof of the theorem.

Corollary 7. One has the following statements:

(i) $\lambda_{1}=\inf \left\{\|\Delta v\|_{p}^{p}, v \in \mathbb{W}_{p}(\Omega)\right.$; $\left.\int_{\Gamma}|\partial u / \partial n|^{p} d s=1\right\}$;

(ii) $0<\lambda_{1} \leq \lambda_{2} \leq \cdots \leq \lambda_{n} \rightarrow+\infty$.
Proof. (i) For $u \in \mathfrak{M}$, set $K_{1}=\{u,-u\}$. It is clear that $\gamma\left(K_{1}\right)=$ $1, \Phi$ is even, and

$$
p \Phi(u)=\max _{K_{1}} p \Phi \geq \inf _{K \in \Gamma_{1}} \max _{K} p \Phi .
$$

Hence

$$
\inf _{u \in \mathfrak{M}} p \Phi(u) \geq \inf _{K \in \Gamma_{1}} \max _{K} p \Phi=\lambda_{1}
$$

On the other hand, for all $K \in \Gamma_{1}$, for all $u \in K$, we have

$$
\sup _{K} p \Phi \geq p \Phi(u) \geq \inf _{u \in \mathfrak{M}} p \Phi(u) .
$$

It follows that

$$
\inf _{K \in \Gamma_{1}} \max _{K} p \Phi=\lambda_{1} \geq \inf _{u \in \mathfrak{M}} p \Phi(u) .
$$

Thus

$$
\lambda_{1}=\inf \left\{\|\Delta v\|_{p}^{p}, v \in \mathbb{W}_{p}(\Omega) ; \int_{\Gamma}\left|\frac{\partial u}{\partial n}\right|^{p} d s=1\right\} .
$$

(ii) For all $i \geq j$, we have $\Gamma_{i} \subset \Gamma_{j}$ and in view of definition of $\lambda_{i}, i \in \mathbb{N}^{*}$, we get $\lambda_{i} \geq \lambda_{j}$. Regarding $\lambda_{n} \rightarrow \infty$, it is proved before in Theorem 6 .

We now turn to the fourth-order nonlinear Steklov boundary eigenvalue problem $\left(E_{p}\right)_{\lambda}$.

Definition 8. One says that a function $u \in \mathbb{W}_{p}(\Omega)$ is weak solution of $\left(E_{p}\right)_{\lambda}$ if

$$
\begin{gathered}
\int_{\Omega}|\Delta u|^{p-2} \Delta u \Delta v d x-\lambda \int_{\partial \Omega}\left|\frac{\partial u}{\partial n}\right|^{p-2} \frac{\partial u}{\partial n} \frac{\partial v}{\partial n} d s \\
=\int_{\Omega}|u|^{p^{*}-2} u v d x \quad \forall v \in \mathbb{W}_{p}(\Omega) .
\end{gathered}
$$

If $u \in \mathbb{W}_{p}(\Omega) \backslash\{0\}$, then it is called an eigenfunction of problem $\left(E_{p}\right)_{\lambda}$.

Lemma 9 (see [19]). Assume that $\Omega$ is a bounded domain of $C^{2}$ in $\mathbb{R}^{N}$.

$$
\text { Then } W^{2, p}(\Omega) \hookrightarrow L^{q}(\Omega) \text { for all } p \leq q \leq N p /(N-2 p) \text {. }
$$
follows.

We formulate our second main result of this paper as

Theorem 10. There exists $\lambda_{1}^{*}<\lambda_{1}$ such that if $\lambda \in\left(\lambda_{1}^{*}, \lambda_{1}\right)$ then $\left(E_{p}\right)_{\lambda}$ admits at least energy solutions $u_{\lambda}$ satisfying

$$
\begin{array}{r}
u_{\lambda} \longrightarrow 0 \quad \text { in } \mathbb{W}_{p}(\Omega), \quad \frac{u_{\lambda}}{\left\|\Delta u_{\lambda}\right\|_{p}} \longrightarrow u^{*} \quad \text { in } \mathbb{W}_{p}(\Omega) \\
\text { as } \lambda \longrightarrow \lambda_{1},
\end{array}
$$

where $u^{*}$ is the first eigenfunction of $\left(E_{p}\right)_{\lambda}^{\prime}$ such that $\left\|\Delta u^{*}\right\|_{p}=$ 1 , associated with the principal eigenvalue $\lambda_{1}$ defined by (11). 
Proof of Theorem 10. Consider the following minimization problem:

$$
\Lambda_{\lambda}=\inf _{u \in \mathbb{W}_{p}(\Omega) \backslash\{0\}} \frac{\|\Delta u\|_{p}^{p}-\lambda \int_{\Gamma}|\partial u / \partial n|^{p} d s}{\|u\|_{p^{*}}^{p}}
$$

The existence of least energy solution follows from the following proposition.

Proposition 11. Assume that $0<\lambda<\lambda_{1}$. If $\Lambda_{\lambda}<S$, then the minimum in (55) is achieved, where $S$ is the Sobolev constant for the embedding $W^{2, p}(\Omega) \hookrightarrow L^{p^{*}}(\Omega)$.

Proof. Let $\left\{u_{m}\right\}_{m \geq 0}$ be a minimizing sequence for $\Lambda_{\lambda}$ such that

$$
\left\|u_{m}\right\|_{p^{*}}^{p}=1
$$

Then,

$$
\left\|\Delta u_{m}\right\|_{p}^{p}-\lambda \int_{\Gamma}\left|\frac{\partial u_{m}}{\partial n}\right|^{p} d s=\Lambda_{\lambda}+o(1) \quad(\text { as } m \longrightarrow \infty) .
$$

Moreover, from (11), we have

$$
\begin{aligned}
\left\|\Delta u_{m}\right\|_{p}^{p} & =\Lambda_{\lambda}+\lambda \int_{\Gamma}\left|\frac{\partial u_{m}}{\partial n}\right|^{p} d s+o(1) \\
& \leq \Lambda_{\lambda}+\frac{\lambda}{\lambda_{1}}\left\|\Delta u_{m}\right\|_{p}^{p}+o(1),
\end{aligned}
$$

which implies that $\left\{u_{m}\right\}$ is bounded in $\mathbb{W}_{p}(\Omega)$. Hence $\left\{\nabla u_{m}\right\}$ is bounded in $W^{1, p}(\Omega)$.

Exploiting the compactness of the embedding $\mathbb{W}_{p}(\Omega) \hookrightarrow$ $L^{p}(\Gamma)$ and $W^{1, p}(\Omega) \hookrightarrow L^{p}(\Omega)$, we deduce that there exists $u \in \mathbb{W}_{p}(\Omega)$ such that

$$
\begin{gathered}
u_{m} \rightarrow u \quad \text { in } \mathbb{W}_{p}(\Omega), \quad \frac{\partial u_{m}}{\partial n} \longrightarrow \frac{\partial u}{\partial n} \text { in } L^{p}(\Gamma), \\
u_{m} \longrightarrow u \quad \text { in } L^{p}(\Omega),
\end{gathered}
$$

up to a subsequence. That is, if we set $v_{m}:=u_{m}-u$, then

$$
\begin{gathered}
v_{m} \rightarrow 0 \quad \text { in } \mathbb{W}_{p}(\Omega), \quad \frac{\partial v_{m}}{\partial n} \longrightarrow 0 \quad \text { in } L^{P}(\Gamma), \\
v_{m} \longrightarrow 0 \quad \text { in } L^{p}(\Omega) .
\end{gathered}
$$

On the other hand, in view of (56), we have $\left\|\Delta u_{m}\right\|_{p}^{p} \geq S$, so that, from (57), we obtain

$$
\lambda \int_{\Gamma}\left|\frac{\partial u_{m}}{\partial n}\right|^{p} d s=\left\|\Delta u_{m}\right\|_{p}^{p}-\Lambda_{\lambda}+o(1) \geq S-\Lambda_{\lambda}+o(1)
$$

which remains bounded away from 0 since $\Lambda_{\lambda}<S$. From this, we deduce that $u \neq 0$.
Now, In view of (59) and (60), we may rewrite (57) as

$$
\|\Delta u\|_{p}^{p}+\left\|\Delta v_{m}\right\|_{p}^{p}-\lambda \int_{\Gamma}\left|\frac{\partial u}{\partial n}\right|^{p} d s=\Lambda_{\lambda}+o(1) .
$$

Moreover, by (56) and Brezis-Lieb Lemma [20], we have

$$
\begin{aligned}
1 & =\left\|u+v_{m}\right\|_{p^{*}}^{p^{*}}=\|u\|_{p^{*}}^{p^{*}}+\left\|v_{m}\right\|_{p^{*}}^{p^{*}}+o(1) \\
& \leq\|u\|_{p^{*}}^{p}+\left\|v_{m}\right\|_{p^{*}}^{p}+o(1) \leq\|u\|_{p^{*}}^{p}+\frac{1}{S}\left\|\Delta v_{m}\right\|_{p}^{p}+o(1),
\end{aligned}
$$

where we use also the fact that both $\|u\|_{p^{*}}$ and $\left\|v_{m}\right\|_{p^{*}}$ do not exceed 1 . Since $\Lambda_{\lambda} \geq 0$ for every $0<\lambda<\lambda_{1}$, the last inequality gives

$$
\Lambda_{\lambda} \leq \Lambda_{\lambda}\|u\|_{p^{*}}^{p}+\frac{\Lambda_{\lambda}}{S}\left\|\Delta v_{m}\right\|_{p}^{p}+o(1) .
$$

By combining this inequality with (62), we obtain

$$
\begin{aligned}
\| \Delta & u \|_{p}^{p}-\lambda \int_{\Gamma}\left|\frac{\partial u}{\partial n}\right|^{p} d s \\
& =\Lambda_{\lambda}-\left\|\Delta v_{m}\right\|_{p}^{p}+o(1) \\
& \leq \Lambda_{\lambda}\|u\|_{p^{*}}^{p}+\left(\frac{\Lambda_{\lambda}}{S}-1\right)\left\|\Delta v_{m}\right\|_{p}^{p}+o(1) \\
& \leq \Lambda_{\lambda}\|u\|_{p^{*}}^{p}+o(1),
\end{aligned}
$$

which shows that $u \neq 0$ is minimizer for (53).

Let $u^{*}$ be a positive eigenfunction of $\left(E_{p}\right)_{\lambda}^{\prime}$ and

$$
\lambda_{1}^{*}=\frac{\left\|\Delta u^{*}\right\|_{p}^{p}-S\left\|u^{*}\right\|_{p^{*}}^{p}}{\left\|\partial u^{*} / \partial n\right\|_{L^{p}(\Gamma)}^{p}}
$$

Thus $\lambda_{1}^{*}<\lambda_{1}$ and, for $\lambda>\lambda_{1}^{*}$, we have

$$
\Lambda_{\lambda} \leq \frac{\left\|\Delta u^{*}\right\|_{p}^{p}-\lambda\left\|\partial u^{*} / \partial n\right\|_{L^{p}(\Gamma)}^{p}}{\left\|u^{*}\right\|_{p^{*}}^{p}}<S .
$$

We now prove the first part of (54). Indeed, in view of the characterization of $u^{*}$ in (53), we have

$$
\begin{array}{r}
\Lambda_{\lambda} \leq \frac{\left\|\Delta u^{*}\right\|_{p}^{p}-\lambda\left\|\partial u^{*} / \partial n\right\|_{L^{p}(\Gamma)}^{p}}{\left\|u^{*}\right\|_{p^{*}}^{p}}=\frac{1-\left(\lambda / \lambda_{1}\right)}{\left\|u^{*}\right\|_{p^{*}}^{p}} \\
\text { as } \lambda \longrightarrow 0
\end{array}
$$

Since $u_{\lambda}$ is a least energy solution of $\left(E_{p}\right)$, we have

$$
\frac{\left\|\Delta u_{\lambda}\right\|_{p}^{p}-\lambda\left\|\partial u_{\lambda} / \partial n\right\|_{L^{p}(\Gamma)}^{p}}{\left\|u_{\lambda}\right\|_{p^{*}}^{p}}=\Lambda_{\lambda}
$$

Moreover, by taking $v=u_{\lambda}$ in (53), we get

$$
\left\|\Delta u_{\lambda}\right\|_{p}^{p}-\lambda\left\|\frac{\partial u_{\lambda}}{\partial n}\right\|_{L^{p}(\Gamma)}^{p}=\left\|u_{\lambda}\right\|_{p^{*}}^{p^{*}}
$$


Identities (69)-(70) readily imply that $\left\|u_{\lambda}\right\|_{p^{*}}=\Lambda_{\lambda}^{(N-2 p) / 2 p^{2}}$. In turn, this and (62) show that

$$
u_{\lambda} \longrightarrow 0 \text { in } L^{p^{*}}(\Omega) \quad \text { as } \lambda \longrightarrow \lambda_{1} .
$$

Moreover, by (69) and (68) we obtain

$$
\left\|\Delta u_{\lambda}\right\|_{p}^{p}-\lambda\left\|\frac{\partial u_{\lambda}}{\partial n}\right\|_{L^{p}(\Gamma)}^{p} \leq \frac{1-\left(\lambda / \lambda_{1}\right)}{\left\|u^{*}\right\|_{p^{*}}^{p}}\left\|u_{\lambda}\right\|_{p^{*}}^{p} .
$$

Thus

$$
\left\|\Delta u_{\lambda}\right\|_{p}^{p} \leq \lambda\left\|\frac{\partial u_{\lambda}}{\partial n}\right\|_{L^{p}(\Gamma)}^{p}+\frac{1-\left(\lambda / \lambda_{1}\right)}{\left\|u^{*}\right\|_{p^{*}}^{p}}\left\|u_{\lambda}\right\|_{p^{*}}^{p} .
$$

Then in view of (11), we get

$$
\left\|\Delta u_{\lambda}\right\|_{p}^{p} \leq \frac{\lambda}{\lambda_{1}}\left\|\Delta u_{\lambda}\right\|_{p}^{p}+\frac{1-\left(\lambda / \lambda_{1}\right)}{\left\|u^{*}\right\|_{p^{*}}^{p}}\left\|u_{\lambda}\right\|_{p^{*}}^{p}
$$

Hence

$$
\left(1-\frac{\lambda}{\lambda_{1}}\right)\left\|\Delta u_{\lambda}\right\|_{p}^{p} \leq \frac{1-\left(\lambda / \lambda_{1}\right)}{\left\|u^{*}\right\|_{p^{*}}^{p}}\left\|u_{\lambda}\right\|_{p^{*}}^{p}
$$

Consequently, using (76), the last inequality implies that

$$
\left\|\Delta u_{\lambda}\right\|_{p}^{p} \leq\left\|u^{*}\right\|_{p^{*}}^{-p}\left\|u_{\lambda}\right\|_{p^{*}}^{p} \longrightarrow 0 \quad \text { as } \lambda \longrightarrow \lambda_{1} .
$$

Finally, we conclude that

$$
u_{\lambda} \longrightarrow 0 \quad \text { in } \mathbb{W}_{p}(\Omega) \quad \text { as } \lambda \longrightarrow \lambda_{1} .
$$

From [21], the inequality

$$
\begin{aligned}
\left|t_{1}-t_{2}\right|^{p} \leq & c\left\{\left(\left|t_{1}\right|^{p-2} t_{1}-\left|t_{2}\right|^{p-2} t_{2}\right) \cdot\left(t_{1}-t_{2}\right)\right\}^{\gamma / 2} \\
& \times\left(\left|t_{1}\right|^{p}+\left|t_{2}\right|^{2}\right)^{1-\gamma / 2},
\end{aligned}
$$

holds true for any $t_{1}, t_{2} \in \mathbb{R}$, with $\gamma=p$ if $1<p<2$ and $\gamma=2$ if $p \geq 2$.

By applying Hölder's inequality, we have

$$
\begin{aligned}
& \left\|\frac{\Delta u_{\lambda}}{\left\|\Delta u_{\lambda}\right\|_{p}}-\Delta u^{*}\right\|_{p}^{p} \\
& =\frac{1}{\left\|\Delta u_{\lambda}\right\|_{p}^{p}}\left\|\Delta u_{\lambda}-\right\| \Delta u_{\lambda}\left\|_{p} \Delta u^{*}\right\|_{p}^{p} \\
& \leq \frac{c}{\left\|\Delta u_{\lambda}\right\|_{p}^{p-1}}\left\{G\left(u_{\lambda}, u^{*}\right)\right\}^{\gamma / 2}\left(\left\|\Delta u_{\lambda}\right\|_{p}^{p}+\left\|\Delta u_{\lambda}\right\|_{p}^{p} \cdot\left\|\Delta u^{*}\right\|^{p}\right)^{1-\gamma / 2} \\
& \leq \frac{c}{\left\|\Delta u_{\lambda}\right\|_{p}^{p \gamma / 2-1}}\left\{G\left(u_{\lambda}, u^{*}\right)\right\}^{\gamma / 2}\left(1+\left\|\Delta u^{*}\right\|^{p}\right)^{1-\gamma / 2},
\end{aligned}
$$

where

$$
\begin{aligned}
& G\left(u_{\lambda}, u^{*}\right) \\
& =\int_{\Omega}\left(\left|\Delta u_{\lambda}\right|^{p-2} \Delta u_{\lambda}-\left|\left\|\Delta u_{\lambda}\right\|_{p} \Delta u^{*}\right|^{p-2}\left(\left\|\Delta u_{\lambda}\right\|_{p} \Delta u^{*}\right)\right) \\
& \quad \cdot\left(\Delta u_{\lambda}-\left\|\Delta u_{\lambda}\right\|_{p} \Delta u^{*}\right) d x \\
& \leq \int_{\Omega}\left|\Delta u_{\lambda}\right|^{p}+\left\|\Delta u_{\lambda}\right\|_{p}\left|\Delta u_{\lambda}\right|^{p-1}\left|\Delta u^{*}\right| \\
& \quad+\left\|\Delta u_{\lambda}\right\|_{p}^{p-1}\left|\Delta u^{*}\right|^{p-1}\left|\Delta u_{\lambda}\right|+\left\|\Delta u_{\lambda}\right\|_{p}^{p}\left|\Delta u^{*}\right|^{p} d x .
\end{aligned}
$$

Then using again Hölder's inequality we obtain

$$
\begin{aligned}
G & \left(u_{\lambda}, u^{*}\right) \\
\leq & \left\|\Delta u_{\lambda}\right\|_{p}^{p}+\left\|\Delta u_{\lambda}\right\|_{p}^{p}\left\|\Delta u^{*}\right\|_{p}^{p} \\
& +\left\|\Delta u_{\lambda}\right\|_{p}^{2 p-1}\left\|\Delta u^{*}\right\|_{p}^{p-1}+\left\|\Delta u_{\lambda}\right\|_{p}^{p}\left\|\Delta u^{*}\right\|_{p}^{p} \\
\leq & \left\|\Delta u_{\lambda}\right\|_{p}^{p}\left(1+\left\|\Delta u^{*}\right\|_{p}^{p}+\left\|\Delta u_{\lambda}\right\|_{p}^{p-1}\left\|\Delta u^{*}\right\|_{p}^{p-1}+\left\|\Delta u^{*}\right\|_{p}^{p}\right) .
\end{aligned}
$$

Hence

$$
\begin{aligned}
& \left\|\frac{\Delta u_{\lambda}}{\left\|\Delta u_{\lambda}\right\|_{p}}-\Delta u^{*}\right\|_{p}^{p} \\
& \leq \mathrm{c}\left\|\Delta u_{\lambda}\right\|_{p}\left(1+\left\|\Delta u^{*}\right\|_{p}^{p}+\left\|\Delta u_{\lambda}\right\|_{p}^{p-1}\left\|\Delta u^{*}\right\|_{p}^{p-1}+\left\|\Delta u^{*}\right\|_{p}^{p}\right)^{\gamma / 2} \\
& \quad \times\left(1+\left\|\Delta u^{*}\right\|^{p}\right)^{1-\gamma / 2} .
\end{aligned}
$$

Finally, we conclude that

$$
\left\|\frac{\Delta u_{\lambda}}{\left\|\Delta u_{\lambda}\right\|_{p}}-\Delta u^{*}\right\|_{p}^{p} \longrightarrow 0 \quad \text { as } \lambda \longrightarrow \lambda_{1}
$$

which proves (54) and then the proof of Theorem 10 is achieved.

\section{Conflict of Interests}

The authors declare that there is no conflict of interests regarding the publication of this paper.

\section{Acknowledgment}

The authors would like to thank the anonymous reviewer for the helpful and constructive comments that greatly contributed to improving the final version of the paper.

\section{References}

[1] J. L. Lions, Quelques Méthodes De Résolution Des problèmes Aux limites Non Linéaires, Dunod, Paris, Farnce, 1969. 
[2] M. W. Steklov, "Sur les problèmes fondamentaux de la physique mathématique," Annales Scientifiques de l'École Normale Supérieure, vol. 19, pp. 455-490, 1902.

[3] F. Gazzola and G. Sweers, "On positivity for the biharmonic operator under Steklov boundary conditions," Archive for Rational Mechanics and Analysis, vol. 188, no. 3, pp. 399-427, 2008.

[4] J. R. Kuttler, "Remarks on a Steklov eigenvalue problem," SIAM Journal on Numerical Analysis, vol. 9, pp. 1-5, 1972.

[5] D. Bucur, A. Ferrero, and F. Gazzola, "On the first eigenvalue of a fourth order Steklov problem," Calculus of Variations and Partial Differential Equations, vol. 35, no. 1, pp. 103-131, 2009.

[6] A. Ferrero, F. Gazzola, and T. Weth, "On a fourth order Steklov eigenvalue problem," Analysis, vol. 25, no. 4, pp. 315-332, 2005.

[7] H. Bi, S. Ren, and Y. Yang, "Conforming finite element approximations for a fourth-order Steklov eigenvalue problem," Mathematical Problems in Engineering, vol. 2011, Article ID 873152, 13 pages, 2011.

[8] E. Berchio, F. Gazzola, and T. Weth, "Critical growth biharmonic elliptic problems under Steklov-type boundary conditions," Advances in Di Erential Equations, vol. 12, no. 3, pp. 381406, 2007.

[9] A. Ferrero and G. Warnault, "On solutions of second and fourth order elliptic equations with power-type nonlinearities," Nonlinear Analysis, Theory, Methods and Applications, vol. 70, no. 8, pp. 2889-2902, 2009.

[10] T. G. Myers, “Thin films with high surface tension," SIAM Review, vol. 40, no. 3, pp. 441-462, 1998.

[11] A. Elkhalil, S. Kellati, and A. Touzani, "On the principal frequency curve of the p-biharmonic operator," Arab Journal of Mathematical Sciences, vol. 17, pp. 89-99, 2011.

[12] A. El Khalil, S. Kellati, and A. Touzani, "A nonlinear boundary problem involving the p-bilaplacian operator," International Journal of Mathematics and Mathematical Sciences, vol. 2005, no. 10, pp. 1525-1537, 2005.

[13] A. Szulkin, "Ljusternick-Schnirelmann theory on $C^{1}$ manifolds," Annales de l'Institut Henri Poincaré, vol. 5, pp. 119-139, 1988.

[14] F. Colasuonno and P. Pucci, "Multiplicity of solutions for $p(x)$ polyharmonic elliptic Kirchhoff equations," Nonlinear Analysis, Theory, Methods and Applications, vol. 74, no. 17, pp. 5962-5974, 2011.

[15] V. F. Lubyshev, "Multiple solutions of an even-order nonlinear problem with convex-concave nonlinearity," Nonlinear Analysis, Theory, Methods \& Applications, vol. 74, no. 4, pp. 1345-1354, 2011.

[16] G. Autuori, F. Colasuonno, and P. Pucci, "On the existence of stationary solutions for higher order p-Kirchhoproblems," Communications in Contemporary Mathematics.

[17] D. Gilbar and N. S. Trudinger, Elliptic Partial Differential Equations of Second Order, Springer, New York, NY, USA, 2nd edition, 1983.

[18] S. L. Trojanski, "On locally convex and diffirenciable norms in certain-separable Banach spaces," Studia Mathematica, vol. 37, pp. 173-180, 1971.

[19] R. Adams, Sobolev Spaces, Academie Press, New York, NY, USA, 1975.

[20] H. Brezis and E. Lieb, "A relation between pointwise convergence of functions and convergence of functionals," Proceedings of the American Mathematical Society, vol. 88, pp. 486-490, 1983.
[21] P. Lindqvist, "On the equation $\operatorname{div}\left(|\nabla u|^{p-2} u\right)+\lambda|u|^{p-2} u=0$," Proceedings of the American Mathematical Society, vol. 109, no. 1, pp. 157-164, 1990. 


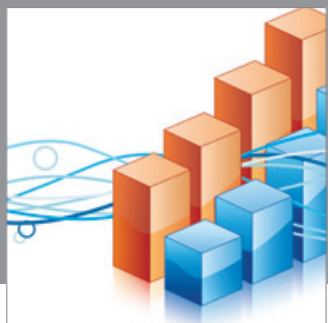

Advances in

Operations Research

mansans

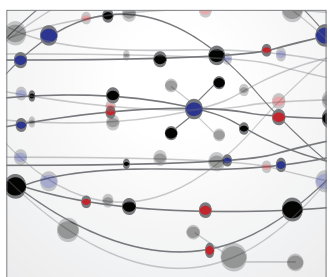

The Scientific World Journal
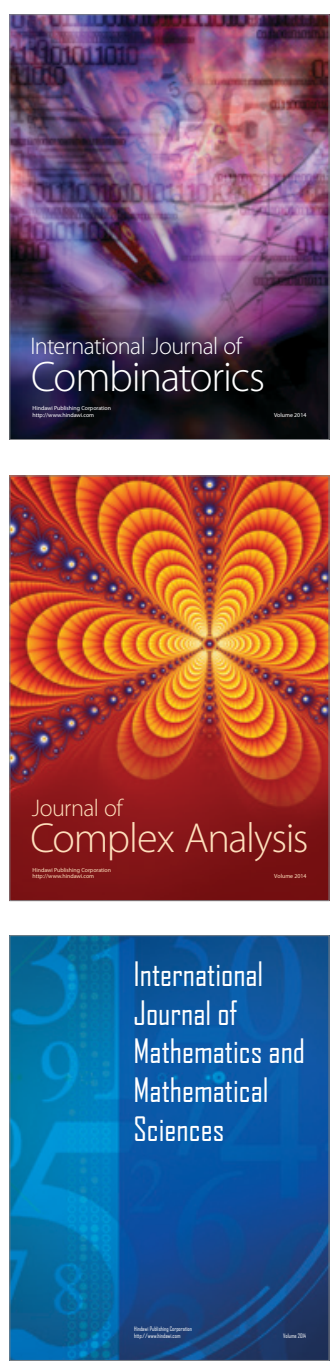
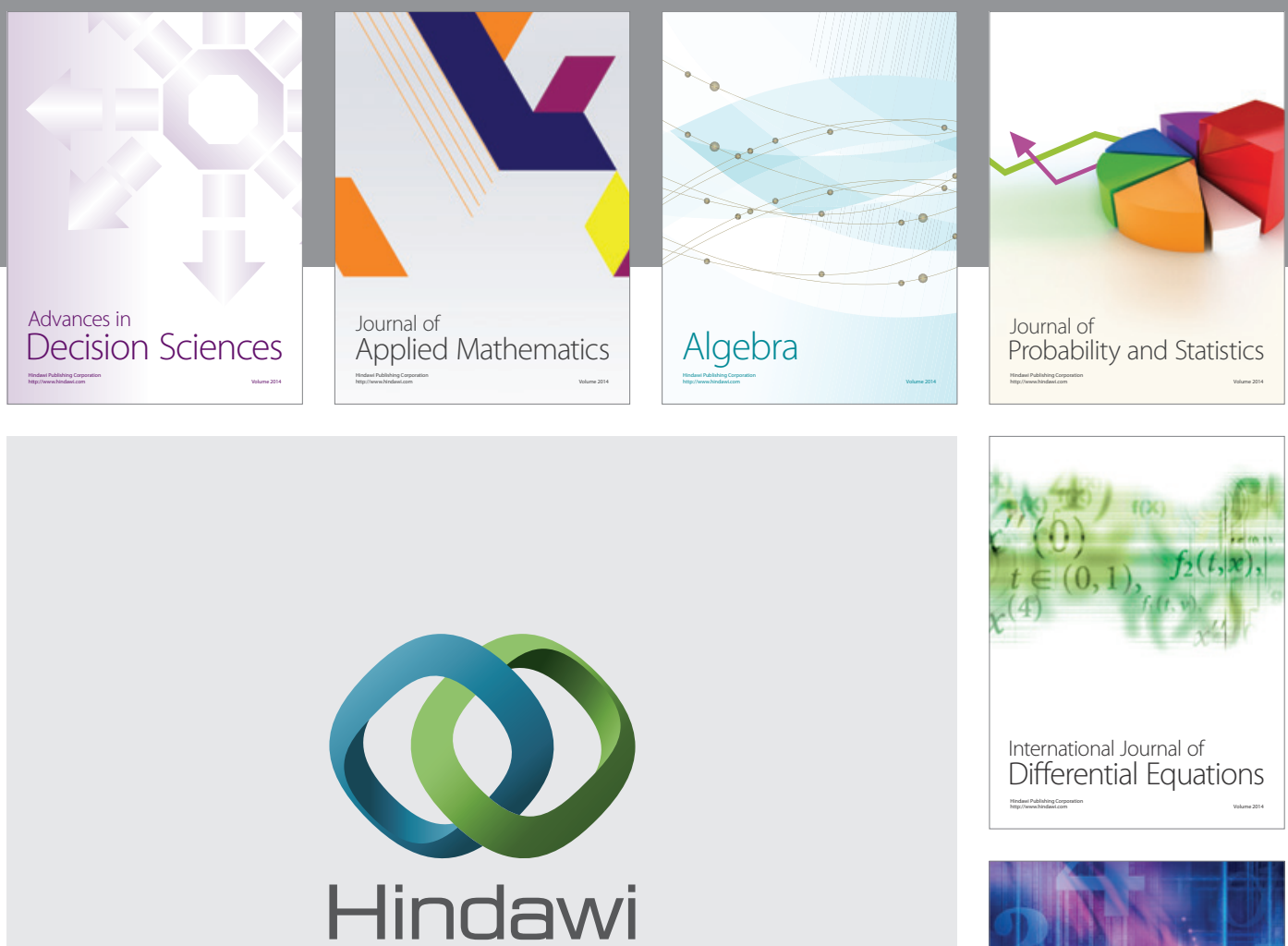

Submit your manuscripts at http://www.hindawi.com
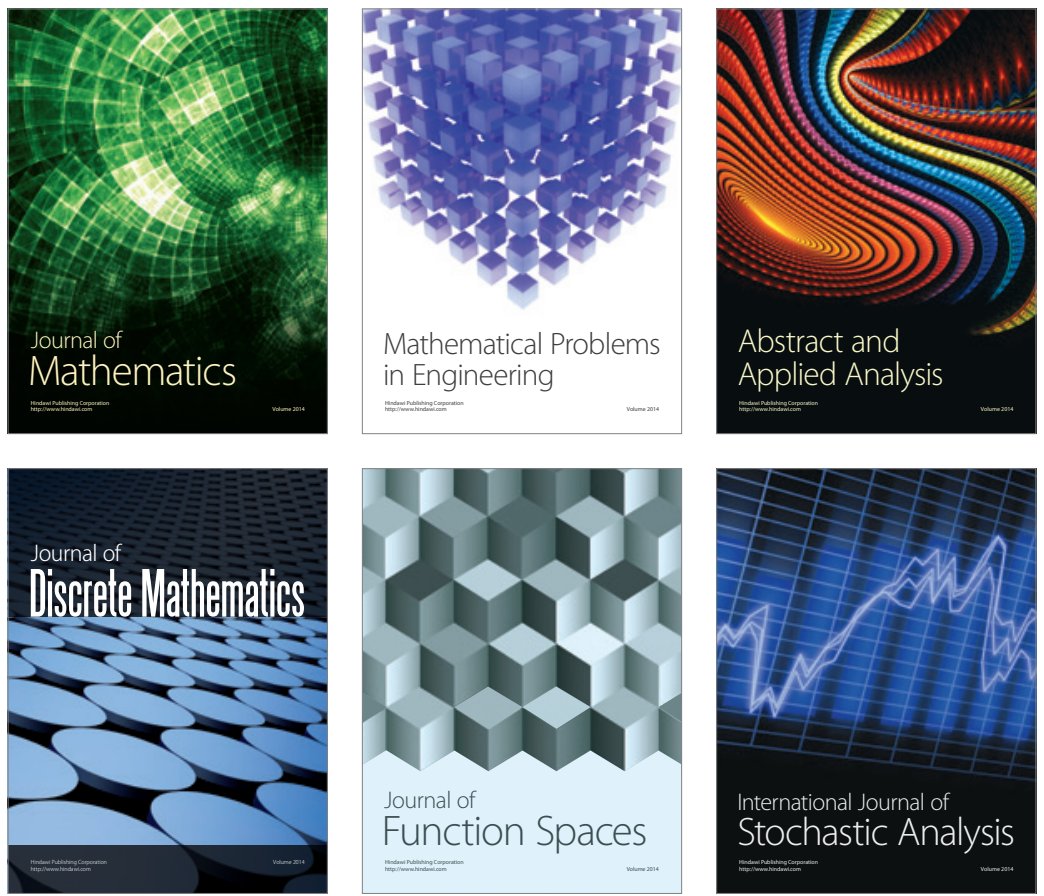

Journal of

Function Spaces

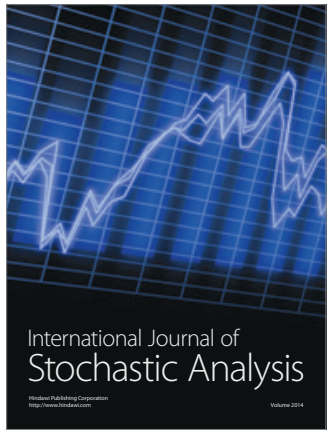

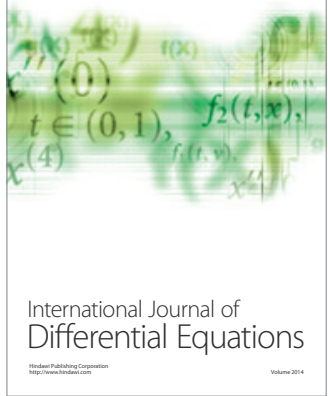
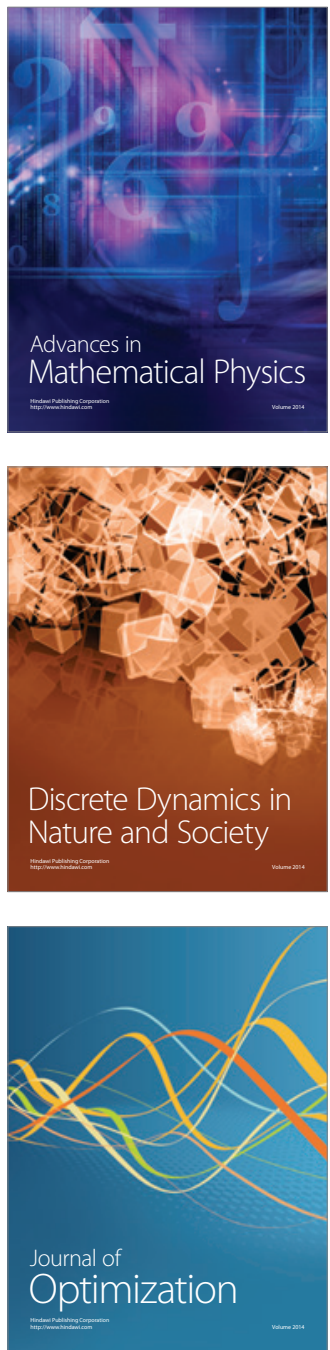